\title{
LIBRAS em saúde: Avaliação na perspectiva de pacientes e de acadêmicos de medicina
}

\author{
Brazilian Sign Language in health: Evaluation from the perspective of patients and medical \\ students \\ Lenguaje De Señas brasileño en salud: Evaluación desde la perspectiva de pacientes y estudiantes \\ médicos
}

\author{
Sidney dos Santos Vasconcelos \\ ORCID: https://orcid.org/0000-0003-2393-7966 \\ Universidade Federal do Pará, Brasil \\ E-mail: sidneyifmsa@gmail.com \\ Gabriel Silva Novais \\ ORCID: https://orcid.org/0000-0002-5420-0337 \\ Universidade Federal do Pará, Brasil \\ E-mail: gbrl10@gmail.com \\ Keya Whitney Weekes \\ ORCID: https://orcid.org/0000-0003-4997-0916 \\ Universidade Federal do Pará, Brasil \\ E-mail: keys121.123@gmail.com \\ Maria Clara Pinheiro da Silva \\ ORCID: https://orcid.org/0000-0001-7697-7406 \\ Universidade Federal do Pará, Brasil \\ E-mail: mariaclarapinheirods@gmail.com \\ Greice de Lemos Cardoso Costa \\ ORCID: https://orcid.org/0000-0002-8916-0283 \\ Universidade Federal do Pará, Brasil \\ E-mail: greice_cardoso@yahoo.com.br \\ Izaura Maria Vieira Cayres Vallinoto \\ ORCID: http://orcid.org/0000-0003-1408-8384 \\ Universidade Federal do Pará, Brasil \\ E-mail: ivallinoto@ufpa.br
}

\begin{abstract}
Resumo
Objetivo: Investigar a ampliação do conhecimento sobre a necessidade do acesso às pessoas com deficiência auditiva aos serviços de saúde. Metodologia: Estudo quantitativo com desenho de estudo descritivo e transversal e faz parte do projeto Libras em saúde da ONG International Federation of Medical Students Association of Brazil, comitê da Universidade Federal do Pará, cujo objetivo geral é investigar o atendimento à pessoa surda na área da saúde na perspectiva acadêmica e, também, do surdo. Resultados: O percentual de avaliações negativas do encontro com profissionais de saúde representou $51 \%$ dentre os bilíngues; e $78 \%$ no grupo de surdos que se comunicam. Foram entrevistados 56 acadêmicos dos terceiro e quarto anos de medicina da UFPA. O pré-teste evidenciou o desconhecimento dos alunos sobre a cultura surda e a língua de sinais. No pós-teste: os alunos conseguiram diferenciar o surdo da pessoa com deficiência auditiva pela inserção ou não na cultura surda. Conclusão: Pacientes surdos utilizam o sistema de saúde de modo diferente de pacientes ouvintes e relatam dificuldades representadas por medo, desconfiança e frustração. $\mathrm{O}$ estudo demonstra que os profissionais da saúde não se encontram preparados para lidar com o atendimento a pessoas com deficiência auditiva, referindo, ainda, a falta de intérpretes para o auxílio nestes atendimentos e a falta de paciência na condução dos procedimentos por meio dos profissionais. Assim, a inclusão do ensino de Libras como sendo disciplina do curso de medicina, se faz um importante instrumento de mudança na realidade social que o deficiente auditivo está incluído atualmente.
\end{abstract}

Palavras-chave: Libras; Saúde; Surdo; Ensino.

\section{Abstract}

Objective: To investigate the expansion of knowledge about the need for access to health services for people with hearing loss. Methodology: This is a quantitative study with a descriptive and cross-sectional study design and is part of the Libras em Saúde project of the NGO International Federation of Medical Students Association of Brazil, committee of the Federal University of Pará, whose general objective is to investigate the assistance to deaf people in 
the health field from an academic perspective and also from the deaf person's perspective. Results: The percentage of negative evaluations of the meeting with health professionals represented $51 \%$ among bilinguals; and $78 \%$ in the group of deaf people who communicate. 56 academics from the third and fourth years of medicine at UFPA were interviewed. The pre-test showed the students' lack of knowledge about deaf culture and Brazilian sign language (Libras). In the post-test: the students were able to differentiate the deaf from the person with hearing loss by insertion or not in the deaf culture. Conclusion: Deaf patients use the health system differently from hearing patients and report difficulties represented by fear, distrust and frustration. The study demonstrates that health professionals are not prepared to deal with the care of people with hearing impairments, also referring to the lack of interpreters to assist in these services and the lack of patience in conducting procedures through professionals. Thus, the inclusion of teaching Brazilian language (Libras) as a discipline of the medical course, becomes an important instrument of change in the social reality that the hearing impaired is currently included.

Keywords: Sign language; Health; Deaf; Teaching.

\section{Resumen}

Objetivo: Investigar la ampliación del conocimiento sobre la necesidad de acceso a los servicios de salud para personas con discapacidad auditiva. Metodología: Se trata de un estudio cuantitativo con un diseño de estudio descriptivo y transversal y es parte del proyecto Libras em Saúde de la ONG Federación Internacional de Asociación de Estudiantes de Medicina de Brasil, comité de la Universidad Federal de Pará, cuyo objetivo general es investigar la atención a las personas sordas en el ámbito de la salud desde una perspectiva académica y también desde la sordera. Resultados: El porcentaje de evaluaciones negativas de la reunión con profesionales de la salud representó el 51\% entre los bilingües; y el $78 \%$ en el grupo de personas sordas que se comunican. Se entrevistó a 56 académicos de tercer y cuarto año de medicina de la UFPA. La prueba preliminar mostró la falta de conocimiento de los estudiantes sobre la cultura de los sordos y el lenguaje de señas. En el post-test: los estudiantes pudieron diferenciar al sordo de la persona con hipoacusia por inserción o no en la cultura sorda. Conclusión: Los pacientes sordos utilizan el sistema de salud de manera diferente a los pacientes oyentes y refieren dificultades representadas por el miedo, la desconfianza y la frustración. El estudio demuestra que los profesionales de la salud no están preparados para atender la atención de personas con discapacidad auditiva, refiriéndose también a la falta de intérpretes para asistir en estos servicios y la falta de paciencia en la realización de trámites a través de profesionales. Así, la inclusión de la enseñanza de Libra como disciplina del curso de medicina, se convierte en un importante instrumento de cambio en la realidad social que actualmente incluye a las personas con discapacidad auditiva.

Palabras clave: Libras; Salud; Sordos; Ensenãnza.

\section{Introdução}

A sociedade brasileira já despertou para as necessidades vitais das camadas sociais que compõem as minorias, neste sentido, entendidas como as que estão à margem da sociedade. Minorias, estas, compostas por sociedades de risco, resultando em exclusão social de caráter não individual, mas, sim, uma situação de privação social coletiva.

Dentre os segmentos que compõem as minorias estão as pessoas desempregadas, os moradores de ruas, os negros, as prostitutas, os portadores de infecções sexualmente transmissíveis e as pessoas com deficiência. Entre os últimos, notadamente, as pessoas surdas, provavelmente, em razão da disseminação do uso da Língua Brasileira de Sinais - Libras, através de movimentos de cunho religioso e universitário.

Para incluir as pessoas surdas na sociedade, foram desenvolvidas, nas últimas décadas, várias legislações com o fito de se estabelecer direitos e obrigações, essas quase sempre imputadas às instituições públicas e privadas. Não obstante, tais legislações, em várias circunstâncias e contextos, não são efetivadas corretamente, o que, consequentemente, acaba por excluir, ainda mais, tal segmento social, ferindo, assim, o fundamento constitucional da dignidade humana. E, assim, comprometendo um dos pilares do atendimento na saúde, a humanização (Brasil, 1998).

O encontro entre profissionais de saúde e pacientes surdos costuma ser marcado por dificuldade na comunicação. A dificuldade de se comunicar com os médicos, de entendê-los e o desconforto no encontro com eles diminuem o número de consultas. Os profissionais dos serviços públicos de saúde, não estão preparados para lidar com as pessoas surdas, que, na grande maioria, não têm domínio da língua portuguesa, o que dificulta, ainda mais, a comunicação entre profissionais e pacientes (Iezzoni, 2004; Zozove \& Doukas, 1994; Ubido, Huntington \& Warburton, 2002). 
Diferentemente da mímica, a Libras constitui uma língua natural, com estrutura gramatical própria em seus níveis fonológico, morfológico, sintático, semântico, capaz de transmitir conceitos concretos e abstratos por meio de canal essencialmente visual (Oliveira, 2012, Quadros, 2004, Karnopp, Quadros \& Quadros, 2004, Bertoloti, 2007). Segundo o capítulo VII do decreto de lei $\mathrm{n}^{\circ}$ 5626/2005 de 22 de dezembro de 2005, os profissionais de saúde devem atender de modo diferencial as pessoas com deficiência auditiva, usuária da Linguagem Brasileira de Sinais (Libras). Contudo, MazzuNascimento et al. 2020 identificaram que dos 5317 cursos de graduação na área da saúde, apenas 2293 (43,1\%) ofereciam disciplina de Libras, sendo a maioria optativa (Mazzu-Nascimento et. AL, 2020). É de extrema importância que todos os profissionais da área da saúde estejam, mesmo que, basicamente, capacitados a atender pessoas com deficiência auditiva, garantindo-lhes um atendimento de qualidade, conseguindo entender e se fazer compreender.

O médico deve ter a habilidade de se comunicar com os pacientes que possuam ou não essas características. O desenvolvimento dessas habilidades está previsto na Resolução $N^{\circ} 4$ do Conselho Nacional de Educação / Conselho de Educação Superior, de 7 de novembro de 2001, que institui as diretrizes curriculares nacionais do curso de graduação em Medicina. Segundo essa resolução, a formação dos médicos tem por objetivo dotar o profissional dos conhecimentos exigidos para o exercício das seguintes competências, entre outras: "comunicar-se adequadamente com os colegas de trabalho, os pacientes e seus familiares"; e "informar e educar seus pacientes, familiares e comunidade em relação à promoção da saúde, prevenção e tratamento, e reabilitação das doenças, usando técnicas apropriadas de comunicação".

No contexto em que muito se discute acessibilidade, torna-se importante difundir conhecimentos sobre a Libras entre discentes de cursos de saúde, para contribuir na formação de profissionais habilitados a compreender e a auxiliar as necessidades das pessoas que a utilizam como sua primeira língua. Para tanto, investigar-se-á a ampliação do conhecimento sobre a necessidade do acesso de pessoas com deficiência auditiva aos serviços de saúde por intermédio de profissionais capazes de estabelecer comunicação e compreender a necessidade desses pacientes além do previsto no modelo biomédico.

\section{Metodologia}

Trata-se de um estudo quantitativo com desenho de estudo descritivo e transversal (Pereira A. S. et al. 2018). A primeira pesquisa foi realizada no CAS (Centro de Atendimento ao Surdo), na cidade de Belém, Estado do Pará, Brasil, com a população de surdos e a segunda pesquisa com os estudantes regularmente matriculados nos terceiro e quarto anos do curso de medicina na Universidade Federal do Pará, em ambiente acadêmico. Participaram 20 surdos cadastrados no CAS (Centro de Atendimento ao Surdo), entre 20 e 50 anos, sendo 12 homens e 08 mulheres, bilingues e que se comunicavam por Libras. Foram incluídos na pesquisa todos os indivíduos de ambos os gêneros e de todas as faixas etárias presentes nas ocasiões das coletas e que concordaram em participar da pesquisa.

Tomaram parte da pesquisa 56 estudantes de medicina, 24 homens e 32 mulheres. Foram incluídos na pesquisa todos os indivíduos de ambos os gêneros e de todas as faixas etárias presentes nas ocasiões das coletas e que concordaram em participar da pesquisa.

A coleta de dados ocorreu entre maio de 2016 e setembro de 2017. Para o estudo com os surdos, os dados foram coletados por meio de entrevista estruturada, contendo formulário preenchido, instrumento que continha questões objetivas relacionadas à comunicação e da relação estabelecida entre estes profissionais e a pessoa surda, com a ajuda de profissional intérprete, o qual busca analisar o atendimento do paciente surdo na visão da pessoa surda. No estudo dos estudantes de Medicina, foi preenchido um formulário objetivo no início do módulo optativo de Libras em maio e junho de 2016 (pré-teste) e um formulário discursivo no final do modulo (pós-teste), que visam quantificar e qualificar os profissionais que estão em formação sobre a necessidade do aprendizado em Libras, o qual analisará o preparo para o atendimento à pessoa surda sob a perspectiva do acadêmico. 
Os dados trabalhados foram apresentados em forma de tabelas e de gráficos por meio do software Microsoft Office Excel 2013.

A pesquisa foi realizada a partir da assinatura de Termo de Consentimento Livre Esclarecido (TCLE) pelos indivíduos participantes. A execução da pesquisa seguiu os aspectos éticos, com aprovação no Comitê de Ética em Pesquisa em seres Humanos do Instituto de Ciências da Saúde.

Este estudo faz parte de um projeto da ONG IFMSA BRAZIL (International Federation of Medical Students Association of Brazil), comitê da Universidade Federal do Pará

\section{Resultados}

Os resultados obtidos pela pesquisa promoveram uma série de conhecimentos sobre a assistência ao surdo na área da saúde, na visão dos próprios surdos e dos discentes. Apresentam-se, a seguir, os resultados obtidos a partir dos questionários realizados com os informantes-chave, utilizando-se formulário para os citados acima.

Na Tabela 1, podem ser vistas as respostas apresentadas pelos surdos a questionamentos realizados acerca do atendimento médico recebido por eles.

Tabela 1 - Questionamentos realizados aos surdos.

\begin{tabular}{llc}
\hline & SIM & NÃO \\
\hline Gosta de ir a consultas médicas & 07 & 13 \\
Vai ao médico com frequência & 04 & 16 \\
Qual frequência (+ de 4 vezes ao ano) & 04 & 16 \\
Entende a receita médica & 07 & 13 \\
Sente seguro em tomar as medicações & 07 & 13 \\
Considera importante o intérprete na consulta & 08 & 12 \\
Existe confusão no seu atendimento com outra doença & 12 & 08 \\
Atendimento ao surdo pelo SUS (má qualidade) & 14 & 06 \\
Tem condições financeiras para um atendimento melhor & 06 & 14 \\
\hline
\end{tabular}

Fonte: Coleta de dados, CAS (2017).

Na Tabela 2 estão descritas as experiências negativas relatadas por alguns dos entrevistados de acordo com as suas formas de comunicação.

Tabela 2 - Total de participantes entrevistados (20) e quantidade de participantes com avaliação negativa.

\begin{tabular}{|c|c|c|}
\hline & Comunicação por Libras & Bilíngue \\
\hline $\begin{array}{l}\text { Percepções diferentes sobre o que se constitui uma comunicação } \\
\text { eficaz (leitura labial, escrita), falta de língua em comum, falta de } \\
\text { paciência }\end{array}$ & 6 & 6 \\
\hline Surdo confundido com deficiente mental & 7 & 2 \\
\hline $\begin{array}{l}\text { Segurança dos medicamentos e outros riscos ocasionados pela } \\
\text { comunicação inadequada }\end{array}$ & 9 & 4 \\
\hline Problemas de comunicação durante o exame físico e procedimentos & 10 & 5 \\
\hline Ausência de intérpretes & 6 & 2 \\
\hline Pouca frequência nas consultas & 9 & 7 \\
\hline
\end{tabular}


A respeito da ausência de intérpretes nos serviços de saúde, seis participantes que se comunicavam por Libras achavam necessária e dois entrevistados bilíngues consideravam importante. Em uma segunda pergunta e observou-se que os surdos valorizam a presença do intérprete, mas com algumas ressalvas: a confiança, o tempo disponível, o constrangimento de se expor frente ao intérprete, sentimentos de piedade (Gráfico 1).

Gráfico 1. Porcentagem de ressalvas ao uso de intérprete no atendimento, do total de 8 respostas positivas dos entrevistados.

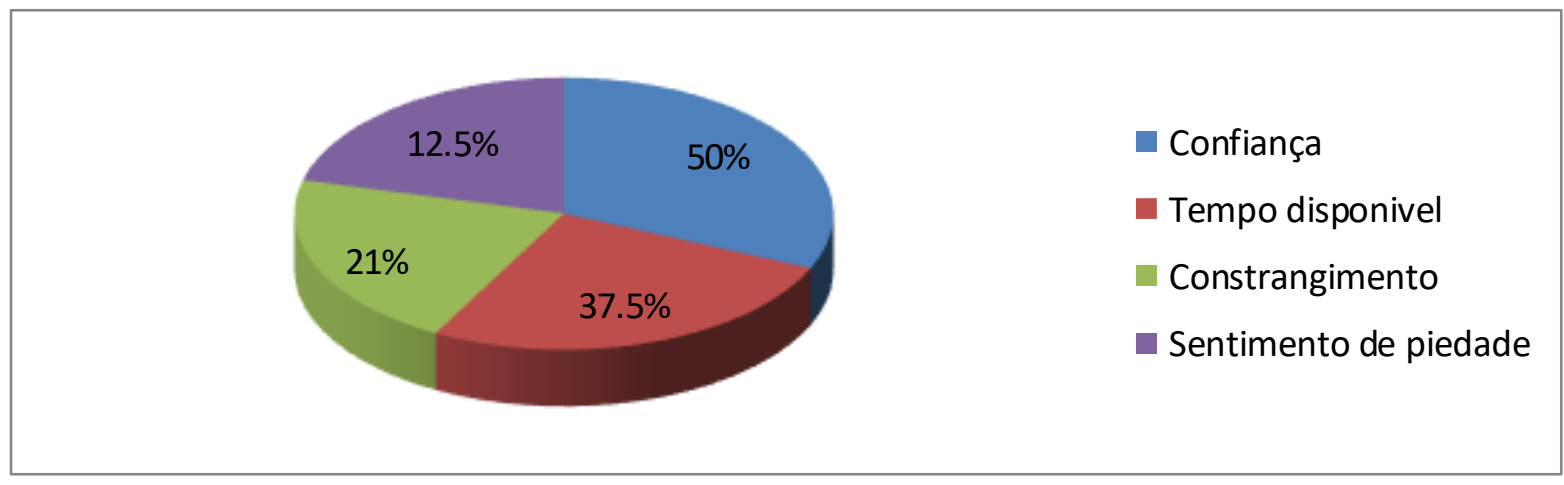

Fonte: Coleta de dados, CAS (2017).

Quanto às queixas inespecíficas, foram obtidas respostas associadas à má vontade do profissional, atendimento de baixa qualidade e dificuldades socioeconômicas como as principais.

Também foram categorizadas as possíveis causas dessas dificuldades, como recursos humanos, por meio da (i) falta de capacitação dos funcionários para o atendimento dessa população e para o uso de insumos tecnológicos, além da ausência de intérpretes já mencionada; e (ii) políticas públicas, devido escassez de profissionais para reabilitação. O percentual de avaliações negativas do encontro com profissionais de saúde representou $51 \%$ dentre os bilíngues e $78 \%$ no grupo de surdos que se comunicam através da língua de sinais.

Foram entrevistados 56 acadêmicos dos terceiro e quarto anos de medicina da UFPA, participantes do curso de Libras, os quais relataram que lhes foram proporcionados saberes que permitirão estabelecer adequadas relações médico-paciente entre eles e os surdos.

O primeiro formulário, denominado pré-teste, evidenciou o desconhecimento dos alunos sobre a cultura surda e a linguagem de sinais, que referiram como mímica e/ou gestos, que a minoria definiu com algo de pouco valor para a comunicação. Os surdos foram identificados, genericamente, como os portadores de deficiência auditiva por todos os participantes (Tabela 3).

Tabela 3 - Conhecimento/desconhecimento dos alunos sobre a cultura surda e a linguagem de sinais.

\begin{tabular}{llll}
\hline & Sim & Não & Total \\
\hline Sabe o que é Libras & 55 & 1 & 56 \\
Estudaram Libras antes & 2 & 54 & 56 \\
Já tiveram contato com surdo durante a graduação (A) & 19 & 37 & 56 \\
Tem familiar surdo (B) & 4 & 52 & 56 \\
Definem surdo como DA & 54 & 2 & 56 \\
Considera Libras mímica ou gesto de pouca importância & 1 & 55 & 56 \\
\hline
\end{tabular}


Dos participantes que citaram já ter contato com surdo (A) e os que têm familiar surdo (B) foi acrescentada pergunta de dificuldade de contato e/ou atendimento. Do grupo (A), 18 tiveram dificuldade de atendimento. Do grupo (B), 3 tiveram essa dificuldade ao atender o paciente surdo. Dos entrevistados, dentre os que responderam que tiveram dificuldade de atendimento, no grupo A, um dos participantes já estudou Libras anteriormente, sendo o único a terminar o nível básico.

No formulário discursivo pós-teste, do curso de Libras como disciplina opcional ao currículo do futuro médico, após as aulas, os vídeos, o contato com intérprete e serem colocados em situações de atendimento fictício a um paciente surdo, a mudança foi significativa: em seus textos, os alunos conseguiram diferenciar o surdo da pessoa com deficiência auditiva pela inserção ou não na cultura surda; destacaram a dificuldade dos surdos em aprender a língua oral por terem a língua de sinais como primeira língua e destacaram a dificuldade de comunicação encontrada tanto pelo paciente quanto para o profissional que desconhece a realidade surda. Do total de alunos entrevistados, (A) 48 diferenciaram surdo de deficiente auditivo; (B) 51 citaram a dificuldade do surdo em aprender a língua oral; (C) 54 relataram a dificuldade de comunicação no atendimento para ambos (profissional e surdo).

Foi relatado que, apesar, de não reconhecerem todos os sinais da Libras, já podem compreender melhor a necessidade do paciente surdo por meio dos componentes da língua de sinais; compreenderam que devem singularizar o processo terapêutico desses indivíduos, ultrapassando o modelo biomédico vigente e valorizar as diferentes nuances que compõem esses pacientes (biopsicossocial e cultural); e enfatizaram a necessidade de proporcionar aos futuros profissionais de saúde noções elementares sobre língua de sinais, por meio de cursos dentro da faculdade.

Os resultados mostram que foi despertado em $55 \%$ dos alunos o interesse de ampliar seus conhecimentos sobre a língua, capacitando-os a atuarem como facilitadores do acesso dos surdos aos serviços de saúde, contribuindo para a garantia de seus direitos como cidadãos. Dos entrevistados, $39 \%$ dos alunos demonstraram que têm interesse em continuar o estudo da língua, porém consideram não ter tempo, enquanto que, $6 \%$ consideraram que o curso básico foi suficiente para o atendimento ao surdo.

\section{Discussão}

A surdez caracteriza-se pela redução da percepção do som, em grau variado, o que dificulta a aquisição da língua oral de forma natural. Aí, então, a comunidade surda passa a usar a língua de sinais como primeiro meio de comunicação, sendo este um dos fatores que despertam o sentimento de pertencimento a uma cultura, embora nem todas as pessoas surdas se considerem membros de uma comunidade, com características únicas, linguagem e normas sociais. Um fato a ser ressaltado é que a surdez se distingue de outras deficiências, não pela deficiência física propriamente dita, mas pela dificuldade de estabelecer comunicação entre pessoas (Fellinger, 2005). Pacientes surdos utilizam o sistema de saúde de modo diferente dos pacientes ouvintes e relatam dificuldades representadas por medo, desconfiança e frustração. Como pode ser visto no presente trabalho, mais da metade dos surdos consideraram o atendimento pelo SUS de má qualidade, seja por falta de recursos, seja por problemas políticos. A consequência é buscarem assistência médica com menos frequência, como visto no resultado da pesquisa, visto que mais da metade da presente amostra não têm condições financeiras para obterem outro tipo de atendimento. Como decorrência desse processo, as pessoas surdas têm pouco conhecimento da assistência em saúde, incluindo menor compreensão dos programas preventivos, incluindo HIV/AIDS (Pagliuca, Aragão \& Almeida, 2007). Conforme os resultados do formulário, um dos maiores obstáculos enfrentados pelos surdos é o de encontrar um serviço de saúde que tenha recursos para atendê-los respeitando a sua deficiência. O relacionamento do surdo com o médico fica extremamente comprometido, pois, eles não conseguem interagir "criar um elo" devido à dificuldade de comunicar-se, sendo uma relação difícil tanto para o surdo como para o profissional que, na maioria das vezes, não estão preparados para atender está clientela (Othero, Dalmaso, 2009). Como regra, nos serviços de saúde não há intérpretes disponíveis, bem como a maioria dos médicos não conhece a 
linguagem de sinais. No presente estudo, considerando a amostra de estudantes investigada, futuros médicos, a quase totalidade relatou não ter habilidade em Libras, refletindo o que é posto na literatura e na realidade acerca dessa lacuna na formação médica e de profissionais da área da saúde (Othero, Dalmaso, 2009). Decorrendo disso, em geral, é o acompanhante do surdo quem explica ao profissional os problemas de saúde que aquele apresenta, sendo, também, a pessoa quem recebe as orientações, ou seja, o paciente surdo não tem oportunidade nem mesmo de expor as suas dúvidas e, muito menos, da individualidade necessária para a sua exposição (Othero, Dalmaso, 2009). A contratação de profissionais intérpretes como parte da equipe de saúde poderia auxiliar a consulta e diminuir a inibição dos pacientes em relação a alguns assuntos (Pereira, Passarin, Nishida \& Garcez, 2020). Os dados obtidos no presente estudo reforçam essa ideia, na medida em que mais da metade dos surdos investigados não entendem a receita médica passada, assim como não se sentem seguros em tomar as medicações receitadas pelo médico. Outra necessidade marcada pelo grupo de surdos entrevistados, diz respeito à necessidade de aceitação da sua condição por parte dos profissionais, não os discriminando como doentes mentais, visto que grande parte dos surdos participantes do estudo relataram a existência de confusão no seu atendimento com o de outras doenças, assim como demandam que os profissionais não sejam indiferentes. Eles querem ser tratados como cidadãos e parte integrante da sociedade. O direito à saúde é um bem fundamental, no entanto, para os surdos, esse direito parece não estar sendo resguardado.

As avaliações feitas no presente trabalho, seja com os surdos, sejam com os acadêmicos, nos leva a afirmar que o problema de comunicação do indivíduo surdo não é orgânico e, sim, social e cultural, pelo fato da sociedade ouvinte não estar preparada para lidar com os surdos e, assim, atender adequadamente suas demandas. No campo da saúde, é possível observar as inúmeras irregularidades existentes e os obstáculos presentes na vida cotidiana da pessoa com deficiência. Entre elas estão o despreparo e a falta de capacitação dos médicos e dos profissionais da saúde em geral, principalmente no que diz respeito ao atendimento, à precariedade dos serviços de saúde prestados, à desigual distribuição de recursos destinados para a área da saúde e às dificuldades enfrentadas pelas pessoas com deficiência em acessibilidade às edificações que prestam serviços na área (Pagliuca, Aragão\& Almeida, 2007). Portanto, vale ressaltar que os serviços de saúde e as equipes de profissionais da saúde devem estar preparados para atuarem de formar a garantir um atendimento digno e de qualidade da saúde dos deficientes (Othero, Dalmaso, 2009). Foi relatada na entrevista a falta de paciência dos médicos no atendimento. Por meio da comunicação estabelecida com o paciente, o profissional pode compreendê-lo como ser holístico e perceber sua visão de mundo, isto é, seu modo de pensar, de sentir e de agir. Dessa forma, poderá entender as necessidades do paciente e, assim, prestar assistência adequada, minimizando seu sofrimento (Pagliuca, Fiúza e Rebolças, 2007). Devido à experiência negativa relatada na pesquisa pelos surdos e a falta de preparo de acordo com os acadêmicos, sugerimos, baseado em literatura, como o médico deve agir no atendimento ao evitar esconder seu rosto e seus lábios com as mãos, com os cabelos e com objetos, da mesma forma com canetas, receitas e máscaras cirúrgicas, devendo, ainda, olhar diretamente para o paciente enquanto fala (Souza \& Porrozzi, 2017). Alguns surdos fazem leitura labial, o que ajuda, grandemente, no processo de comunicação médicopaciente, porém, quando o médico não atenta para o fato de que esconder a boca impede que isto ocorra, surge mais uma barreira que dificulta essa comunicação. Muitas vezes, o surdo não consegue ler o que o médico escreve porque existem palavras difíceis, termos técnicos, porque o surdo não conhece bem o português ou porque a letra do médico é ilegível (Pereira, Passarin, Nishida \& Garcez, 2020). Utilizar a escrita pode ser proveitoso durante a entrevista médica para a comunicação com esses pacientes. No entanto, é importante lembrar que, para explicações complexas, é comum a população surda ter menos instrução que a população em geral (Santos \& Shiratori; 2004). Sendo assim, eles necessitam de maior atenção e de cuidados quando utilizam termos técnicos para as explicações. É importante lembrar que uma letra legível evita confusões no entendimento e evita, também, erros em medicação (Santos \& Shiratori; 2004). Embora a Língua Brasileira de Sinais e os outros recursos de expressão a ela associados sejam reconhecidos como meio legal de comunicação e de expressão, não é 
obrigatório que os médicos saibam ou entendam Libras. Por outro lado, as pessoas surdas que se comunicam usando a língua de sinais, têm gramática e vocabulário diferentes da língua portuguesa; dessa maneira, uma pessoa que nasceu surda no Brasil, pode ser fluente em Libras e não em português (Chaveiro, Barbosa \& Porto, 2008). Em algumas situações o surdo entende o profissional: quando o profissional usa Libras, quando escreve fácil, quando fala devagar e quando o surdo lê lábios. O encontro com o paciente surdo pode ser esporádico, mas o desafio para o médico está além dos serviços especializados. Habilidades no trabalho com pessoas que não partilham a língua oral e apresentam cultura própria não são rotineiramente ensinadas. Por isso, o médico pode não estar preparado para o encontro com o surdo (Porter, 1999). De acordo com os dados da pesquisa com surdos e acadêmicos, concluímos que se o médico dominasse a Libras, o problema da comunicação acabaria. Dado semelhante foi encontrado em outra pesquisa na literatura, em que foi identificada, dentre as propostas dos surdos, a necessidade de profissionais que saibam usar Libras (Barbosa, 1999)). A interação médico-paciente é constituída por processos complexos, utilizando ferramentas verbais e não verbais para que ocorra a troca de informações entre medico e paciente, tais interações podem interferir no diagnóstico e na adesão ao plano terapêutico (Pereira, Passarin, Nishida \& Garcez, 2020). Quando o surdo busca atendimento de saúde e está com sinais clínicos de doença, é mais fácil para o médico detectar o que está ocorrendo; porém, em doenças sem causa aparente, em que é necessário coletar, de modo ampliado, a história do paciente, a situação torna-se mais complicada. A proposta que se torna necessária é que os médicos, principalmente aqueles componentes da Atenção Básica e da Saúde da Família, sejam capacitados para se comunicarem de maneira eficiente com tais clientes. Essa capacitação se concretizaria na oferta de cursos de Libras (Língua Brasileira de Sinais) a todos os profissionais de Saúde de tais áreas, para que, pelo menos, esses profissionais tenham um mínimo de entendimento necessário para o atendimento do paciente (Chaveiro \& Barbosa, 2005).

A falta de intérpretes de Libras constitui uma barreira nas instituições de saúde do Brasil, o que torna, ainda, mais complicada a vida das pessoas com surdez que procuram atendimento ou que solicitam ajuda nestas instituições (Barbosa, 2003). A não compreensão de profissionais sobre as particularidades dos surdos, sejam em relação à identidade da pessoa surda e seus fatores culturais, essa problemática fragiliza a relação entre o profissional e paciente (Pereira, Passarin, Nishida \& Garcez, 2020). Não se concebe instituições que não ofereçam ao surdo, intérpretes e profissionais capazes de comunicar-se com eles, da mesma forma que não se concebe instituições que não tenham rampas ou elevadores. Não adaptar às necessidades dos grupos minoritários é um fator de exclusão social. Em relação à percepção dos surdos quanto à presença dos intérpretes de Libras, na coleta de dados, observou-se que os surdos valorizam a presença do intérprete, mas com algumas ressalvas: a confiança, o tempo disponível, o constrangimento de se expor frente ao intérprete, os sentimentos de piedade. A presença do intérprete de Libras para surdos é essencial nos espaços da área da saúde, mas não prepara o médico para uma inclusão efetiva. No dia em que o tradutor falta, não está disponível para acompanhar os surdos, ou mesmo até quando se faz presente, pode dificultar a interação: médico-paciente, embora a contratação do profissional intérprete seja correta, ressente-se a ausência de uma reflexão mais aprofundada sobre a diversidade e sobre uma verdadeira perspectiva da inclusão (BARBOSA, 2003). A comunicação, tanto para surdos quanto para pessoas sem deficiência, é indicativo de qualidade de vida, portanto, quando os médicos sabem comunicar-se com os surdos, promovem uma assistência na área de saúde humanizada e focalizada no contexto de uma sociedade inclusiva. Para o médico, uma efetiva comunicação com seus clientes propicia um atendimento de melhor qualidade, portanto a sua capacitação para atender a esses pacientes, é uma necessidade urgente, uma formação que contemple os métodos de comunicação, a cultura surda, as noções básicas de Libras e como se posicionar frente ao atendimento do surdo assegura o acesso aos cuidados de saúde (Witte \& Kuzel, 2000; Glat \& Pletsch, 2010, Pereira, Passarin, Nishida \& Garcez, 2020). Através dos dados coletados, concluímos que a elaboração do curso de Libras em saúde despertou, nos participantes, a percepção e o desejo de ampliar os conhecimentos sobre a Libras e contribuir para minimizar a desinformação provocada pelas barreiras linguística e cultural existentes entre ouvintes e surdos. Ofertou-se aos discentes a oportunidade de adquirir os 
conhecimentos mínimos sobre as Libras, qualificando-os à atuação profissional e para que o contato com os pacientes surdos seja uma demonstração de inclusão social. O grande desafio das universidades é formar profissionais que não sejam, apenas, instrumentos de transmissão de conhecimentos, mas, sobretudo, de novas atitudes e práticas que valorizem a diversidade humana.

\section{Conclusão}

A execução do presente estudo representou uma demonstração de inclusão social do surdo na Faculdade de Medicina, enquanto protagonista, possibilitando à comunidade surda se manifestar para expor à comunidade acadêmica suas demandas específicas para um atendimento de saúde adequado. De igual forma, oportunizou, a cinquenta e seis alunos regularmente matriculados, a aquisição de conhecimento mínimo acerca de Libras para fins de atendimento médico, o que tornou estes acadêmicos futuros profissionais qualificados ao atendimento do paciente surdo.

A conquista de uma prática em saúde inclusiva, em relação à pessoa surda, será alcançada quando noções básicas sobre língua de sinais e as particularidades culturais e linguísticas da comunidade surda forem considerados conhecimentos indispensáveis que devem ser ministrados, ainda, no transcorrer da formação acadêmica dos profissionais, em todas as suas variáveis e extensão. Dessa forma, as habilidades comunicativas necessárias à satisfatória assistência a essa população serão desenvolvidas e praticadas, favorecendo as relações interpessoais.

A comunicação verbal utilizada pelos médicos não é suficiente para estabelecer o vínculo, sendo, portanto, ineficaz, e podendo levar a erros no diagnóstico das doenças e no tratamento. Com o domínio dessa língua, o médico conseguirá estabelecer uma comunicação de qualidade e identificar as dificuldades, os sintomas e as situações as quais são passadas pelo paciente, sentindo-se mais à vontade, disposto e identificado com o profissional, demonstrando a importância da inclusão social e respeito pelo cidadão portador de deficiência e executando as demais habilidades às quais o acadêmico de medicina é dotado a ter em toda a sua formação. Com esses ajustamentos às diferentes habilidades mantenedoras da relação médicopaciente surdo, certamente, será revertido o indicador relativo à procura em baixa frequência por este usuário ao serviço de saúde.

A construção de uma prática em saúde que transforme a assistência à pessoa surda irá acontecer por meio de estudos de ações e de vontade política; assim, será possível reorganizar o atendimento, 1) com a afirmação da presença de intérpretes nos serviços de saúde para assegurar ao paciente surdo à decisão de utilização do auxílio do intérprete ou não; 2) com a garantia de programas de educação em saúde a esse perfil de usuário da rede, para assegurar e ampliar o conhecimento acerca de sua própria saúde; 3) com a capacitação dos médicos já vinculados à Atenção Básica e à Saúde da Família para suprimento imediato; 4) dar conhecimento não somente aos acadêmicos de medicina, mas a todos da área da saúde, acerca das demandas dos surdos quanto à comunicação com os profissionais da saúde por ocasião da assistência; 5) capacitar os acadêmicos não somente da medicina, mas da área da saúde da necessidade de desenvolvimento de habilidades de comunicação e estimular novas e futuras experiências vivenciadas com o curso de Libras.

Entendendo a complexidade e a atualidade do tema, bem como as constantes mudanças ocorridas no currículo médico exigido das faculdades de medicina, trabalhos como este devem ocorrer de forma transversal e contínua. Entender se os novos profissionais de saúde estão aprendendo e como estão aprendendo é uma contribuição vital para a saúde pública de qualidade. A fim de melhorar e fazer-se exercer a equidade dentro dos sistemas de saúde.

\section{Agradecimentos}

Os autores agradecem aos surdos do Centro de Atendimento ao Surdo e aos estudantes do curso de Medicina da 
Research, Society and Development, v. 10, n. 8, e15510816225, 2021

(CC BY 4.0) | ISSN 2525-3409 | DOI: http://dx.doi.org/10.33448/rsd-v10i8.16225

Universidade Federal do Pará pela contribuição ao estudo e à Faculdade de Medicina da Universidade Federal do Pará.

\section{Referências}

Brasil, Governo Federal (1998). Constituição da república Federativa do Brasil. Senado Federal.

Lisa, I. I., et al (2004). Communicating about health care: observations from persons who are deaf or hard of hearing. Annals of Internal Medicine, 140 (5)356362 .

Zozove, P., \& Doukas, D. J. (1994). Health Care for Decfcand Hard-of-Hecaring Persons. Fam Med, $26387-90$.

Ubido, J.; et al (2002). Inequalities in access to healthcare faced by women who are deaf. Health \& social care in the community, 10 (4) $247-253$.

Oliveira, F. B. (2012). Desafios na inclusão dos surdos e o intérprete de Libras. Revista Diálogos \& Saberes, 8 (1)

Quadros, R. M. (2004). O tradutor e intérprete de língua brasileira de sinais e língua portuguesa. SEESP.

Karnopp, E. Q., \& Quadros, R. M. De (2004). Língua de sinais brasileira - estudos linguisticos. Artmed.

Bertoloti, R. T (2007). Libras como possibilidade e alternativa para o ensino da Língua Portuguesa para o aluno surdo. Universidade Estadual do Oeste do Paraná.

Mazzu-Nascimento, T., et al (2020). Fragilidade na formação dos profissionais de saúde quanto à Língua Brasileira de Sinais: reflexo na atenção à saúde dos surdos. Audiol, Commun. Res., 25

Fellinger, J.., et al (2005). Mental distress and quality of life in a deaf population. Social Psychiatry and Psychiatric Epidemiology, 40 (9) 737-742.

Pagliuca, L. M. F., et al (2007). Acessibilidade e deficiência física: identificação de barreiras arquitetônicas em áreas internas de hospitais de Sobral, Ceará. Revista da Escola de Enfermagem da USP, 41 (4) 581-588.

Othero, M. B., \& Dalmaso, A. S. W. (2009). Pessoas com deficiência na atenção primária: discurso e prática de profissionais em um centro de saúdeescola. Interface-Comunicação, Saúde, Educação 13 (28).

Pereira, A. A. C., et al (2020). "Meu Sonho É Ser Compreendido: Uma Análise da Interação Médico-Paciente Surdo durante Assistência à Saúde. Rev. Bras. De Educação Médica. 44 (4)

Pagliuca, L. M. F., et al (2007). Aspectos da comunicação da enfermeira com o deficiente auditivo. Revista da Escola de Enfermagem da USP, 41 (3)

Souza, M. T., \& Porrozzi, R. (2017). Ensino de Libras para os profissionais de saúde: uma necessidade premente. Revista Práxis, 1 (2)

Santos, É. M., \& Shiratori, K. (2004). As necessidades de saúde no mundo do silêncio: um diálogo com os surdos. Revista Eletrônica de Enfermagem, 6 (1)

Chaveiro, N., et al (2008). Literature revision about the attendance of deaf patient by health professionals. Revista da Escola de Enfermagem da USP, 42 (3) $578-583$.

Porter, A. (1999). Sign-language interpretation in psychotherapy with deaf patients. American Journal of Psychotherapy, 53 (2) 163-176,

Barbosa, M. A., et al (1999). Ensino e saúde: o que pensam e o que sabem os deficientes auditivos. Revista Eletrônica de Enfermagem, 1 (1)

Chaveiro, N. \& Barbosa, M. A. (2005). Assistência ao surdo na área de saúde como fator de inclusão social. Revista da Escola de Enfermagem da USP, 39 (4)

Barbosa, M. A., et al (2003). Língua Brasileira de Sinais: um desafio para a assistência de enfermagem. Rev. enferm. UERJ, 11 (3) $247-251$.

Witte, T. N., \& Kuzel, A. J. (2000). Elderly deaf patients' health care experiences. The Journal of the American Board of Family Practice, 13 (1) 17-22

Glat, R., \& Pletsch, M. D. (2010). O papel da Universidade no contexto da política de Educação Inclusiva: reflexões sobre a formação de recursos humanos e a produção de conhecimento. Revista Educação Especial, 23 (38)

Pereira, A. S., et al. (2018). Metodologia da pesquisa científica. UFSM 